\title{
SAÚDE DA MULHER NEGRA BRASILEIRA: A NECESSÁRIA INTERSECÇÃO EM RAÇA, GÊNERO E CLASSE
}

\section{Brazilian black woman health: the necessary intersection in race, gender and class}

Silvana Silva do Nascimento (UFPE)

Informações do artigo

Recebido em 11/03/2018

Aceito em 21/04/2018

doi> 10.25247/2447-861X.2018.n243.p91-103

\begin{abstract}
Resumo
O presente estudo objetiva discutir a saúde da mulher negra e suas particularidades, considerando a necessária intersecção entre raça, gênero e classe. Norteadas pela perspectiva histórica, o percurso metodológico adotado contou com uma pesquisa exploratória, composta por levantamento bibliográfico e documental. Os resultados do estudo apontam que $51,6 \%$ da população brasileira é composta por mulheres. Conforme o IPEA (2013) as mulheres negras representavam $50 \%$ da população feminina do país. Em Recife $64 \%$ da população feminina que se autodenomina negra. A Política Nacional de Saúde Integral da População Negra (2006) apresenta que essa população possui particularidades biológicas que os tornam mais suscetíveis a algumas doenças. Verificamos, também, que, conforme os dados coletados, "a pobreza no Brasil tem raça/cor, sexo e etnia. A "feminização da pobreza" está associada às condições de vida, considerando os determinantes sociais, econômicos, políticos, culturais que contribuem para que a mulher negra seja mais suscetível a algumas doenças.
\end{abstract}

Palavras-Chave: Raça. Gênero. Pobreza. Saúde.

\section{Abstract}

The present study aims to discuss the health of black women and their particularities, considering the necessary intersection between race, gender and class. Based on the historical perspective, the methodological approach adopted was an exploratory research, made up of a bibliographical and documentary survey. The results of the study indicate that $51.6 \%$ of the Brazilian population is women, according to IPEA (2013), black women represented $50 \%$ of the country's female population. In Recife $64 \%$ of the female population that calls itself black. The National Policy of Integral Health of the Black Population (2006) shows that this population has biological peculiarities that make them more susceptible to some diseases. We also verified that, according to the data collected, "poverty in Brazil has race / color, sex and ethnicity. The "feminization of poverty" is associated with the conditions of life - the social, economic, political, and cultural determinants that contributes to the black woman being more susceptible to some diseases.

Keywords: Gender. Race. Gender. Poverty. Health. 


\section{Preconceito de cor, raça e origem do racismo no Brasil}

O preconceito de cor, segundo Guimarães (2008), tem origem no século XVI, no início do processo de expansão territorial, quando grande parte dos europeus teve seu primeiro contato com a população africana. O autor afirma ainda que, segundo relatos, o que mais chamou a atenção dos europeus nos primeiros contatos com africanos foi a cor da pele dos povos dominados. Ressalta-se que, nesse período, o povo europeu já era guerreiro e hierarquizado e teorizava sobre a inferioridade dos povos conquistados. [...] daí brota uma primeira fonte de sentimento preconceito, pois, no simbolismo das cores, no ocidente cristão, o negro significa a derrota, a morte, o pecado, enquanto o branco significa o sucesso, a pureza e a sabedoria [...] (GUIMARÃES, 2008,12).

Essa ideologia foi reproduzida no processo de escravidão da população negra no Brasil. Nessa condição, homens e mulheres eram equiparados a coisa ou mercadoria, sujeito (a) à propriedade e ao domínio de outrem e, portanto, privado de quaisquer direitos civis. Cabia ao senhor dono do escravo - homem branco, europeu, colonizador e detentor da riqueza fruto de uma sociedade patriarcal - o direito de utilizar o escravo do modo que melhor Ihe conviesse, tirando-Ihe o maior proveito possível. Ou seja, torna-se explicito que o racismo ou preconceito de cor surge no país em um contexto histórico determinado, quando a população negra é vitimada pela escravidão.

Outro elemento pertinente é a definição de raça, que contribui fortemente para a perpetuação do preconceito. A expressão raça se consolidou nos meios acadêmicos e na fala popular com significado biológico no século XIX; o termo "raça negra" passou a ser mencionado com sentido biológico, mas a fim de marcar a inferioridade atribuída à população negra (GUIMARÃES, 2008). Contudo, Oliveira (2003) afirma a inexistência de um conceito universal sobre o que seja raça.

A palavra raça e o conteúdo histórico desse conceito refere-se a algo que não existe. Raça nada mais exprime do que o entendimento que a ideologia racista quer que ela possua. Ao passo que etnia é uma palavra e um conceito que incorpora a condição biológica humana sem as gradações de superioridade e inferioridade, hierarquização que é inerente à raça dos racistas, ao mesmo tempo em que agrega o ambiente físico e cultural, em todas as suas dimensões (OLIVEIRA, 2003,54).

Munanga (2000) parte do mesmo entendimento de Oliveira (2003) e afirma que raça é um conceito carregado de ideologia e, como todas as ideologias, oculta algo e, nesse caso, 
é a relação de poder e de dominação. O campo semântico do conceito de raça é determinado pela estrutura global da sociedade e pelas relações de poder que a governam. Contudo, a continuidade do uso do termo em estudos nas áreas das ciências sociais se justifica ao considerar a realidade social e política onde a raça é considerada como uma construção sociológica e uma categoria social de dominação e de exclusão (MUNANGA, 2000).

Nesse contexto, o racismo se caracteriza pela crença na existência das raças naturalmente hierarquizadas; consiste em considerar que os atributos intelectuais e morais de um dado grupo são consequências diretas de suas características físicas ou biológicas (MUNANGA, 2000).

De acordo com Santos (2005), existe, no Brasil, a cultura política da negação do racismo. A autora utiliza como referência a pesquisa da Fundação Perseu Abramo, Discriminação Racial e Preconceito de Cor no Brasil (2003). A pesquisa revela que $89 \%$ das pessoas entrevistadas reconhecem o Brasil como um país racista, porém $87 \%$ dos brasileiros dizem nunca ter-se sentido discriminado. A pesquisadora alerta que os preconceitos e as discriminações são assimilados no contexto histórico da formação brasileira e são aplicadas no cotidiano seja na esfera pessoal como institucional.

Tal situação a autora atribui às consequências deixadas pelo regime de escravidão que submeteu a mão de obra africana a trabalhos forçados, valendo-se de mecanismos de repressão e do falso valor atribuído ao que é ser negro. Esse falso valor repercute até os dias atuais sobre a população negra, no que se refere a sua estética e a sua intelectualidade, como forma de garantir a subalternidade (SANTOS, 2005).

Tal realidade é constatada diante dos dados que apontam a população negra ocupando piores indicadores na saúde, educação, renda, ocupação, habitação, entre outros, posicionando-se nas escalas mais baixas da sociedade. Essa condição de vulnerabilidade tem rebatimentos diversos no processo saúde-doença da população negra brasileira.

\section{Raça, gênero, classe e saúde da mulher negra}

Analisar a saúde da mulher negra no contexto histórico brasileiro requer a reflexão dos fatores sociais que impactam a vida dessa população, ou seja, torna-se imperativo que a abordagem da temática seja realizada de modo transversal, considerando a intersecção entre as categorias de análise, raça, gênero e classe, demarcando a problematização das opressões 
presentes na perpetuação da violência contra as mulheres. A interseccionalidade foi identificado como ferramenta teórico-metodológica fundamental para o entendimento das dinâmicas sociais que impactam na vida das mulheres, o termo é definido como uma conceituação do problema que busca capturar as consequências estruturais e dinâmicas da interação entre dois ou mais eixos da subordinação (CRENSHAW, 2002).

Nesse contexto, ao pensar o debate acerca da saúde, torna-se pertinente lembrar que o conceito ampliado de saúde adotado pela Constituição Federal Brasileira, promulgada em 1988, legitimou o conceito de Saúde definido na 8a Conferência Nacional de Saúde: a saúde passou a ser entendida como "a resultante das condições de alimentação, habitação, educação, renda, meio ambiente, trabalho, emprego, lazer, liberdade, acesso e posse de terra e acesso a serviços de saúde" (BRASIL, 1986). Ou seja, podemos concluir que há uma clara inter-relação entre saúde, condições de vida e as relações sociais que se estabelecem e se desenvolvem ao logo da vida.

A saúde não poderia mais ser entendida como mera ausência de doenças, daí a necessidade de considerar a transversalidade com aspectos como a pobreza, desigualdade, racismo e violência, bem como as particularidades de gênero.

E refletir as particularidades que perpassam a saúde da população negra requer lembrar as particularidades biológicas que a tornam mais sensível a algumas doenças e, além disso, não podemos desconsiderar as doenças oriundas das precárias condições de vida. Tal temática reveste-se de importância ao considerarmos que ainda são escassas as produções teóricas/acadêmicas que abordam as disparidades sociais como fator relevante no que se refere aos cuidados em saúde. Contudo, destaca-se a necessidade do enfoque étnico racial nos estudos da saúde, como forma de reconhecimento da discriminação histórica sofrida pela população negra.

Nesse sentido, conhecer os perfis de morbimortalidade da população brasileira se torna necessário para subsidiar a objetivação de políticas e ações de saúde universais destinadas à superação de iniquidades de grupos específicos. Assim, a inserção do quesito raça/cor nos sistemas de informações de saúde contribuiu para evidenciar mediante constatações empíricas, diferenciais raciais e vulnerabilidades produzidas no processo saúdedoença (CUNHA, 2012).

A relevância se agiganta quando nos deparamos com as estatísticas que apontam o quantitativo da população negra brasileira. O Instituto Brasileiro de Geografia e Estatística - 
IBGE (2017), aponta que a 55,1 \% da população brasileira se autodeclara como preta ou parda. De acordo com o Instituto de Pesquisa Econômica Aplicada-IPEA (2013), as mulheres representam $51,6 \%$ da população brasileira e as negras representam $50 \%$ da população feminina do país. Ao mencionar o Nordeste, esse número chega a 69,9\%. No Recife, segundo o Instituto, o percentual de mulheres negras chega a 64\% da população feminina recifense.

Brasil (2005) diz que, apesar da dificuldade em implementar a variável cor ("quesito $\left(\operatorname{cor}^{\prime \prime}\right)^{1}$ em todos os sistemas de informação da área de saúde, fato que impede uma análise mais consistente da saúde da mulher negra, é possível evidenciar que,

A taxa de analfabetismo é o dobro das brancas; são majoritariamente chefes de família sem cônjuge e com filhos; por razões sociais ou de discriminação, as mulheres negras têm menor acesso aos serviços de saúde de boa qualidade, à atenção ginecológica e à assistência obstétrica - seja no prénatal, parto ou puerpério; e maior risco que as brancas de contrair e de morrer mais cedo de determinadas doenças (BRASIL, 2005, 7).

Identifica-se que $40 \%$ dos lares brasileiros são chefiados por mulheres (IPEA, 2015). Em Pernambuco, as mulheres negras são chefes de 65\% das famílias e as mulheres brancas, de $35 \%$. Dos lares chefiados por mulheres negras no estado, $77,2 \%$ têm renda domiciliar de até um salário mínimo (SANTOS, 2014). Se partimos para a esfera dos programas sociais, contatamos que, dentre as famílias atendidas pelo Programa Bolsa Família, 93\% são chefiadas por mulheres e $73 \%$ dos cadastrados no Bolsa Família são pretos ou pardos autodeclarados (MARTINS, 2014).

Frente a tal informação verifica-se que é inevitável a conexão entre a miséria e a recorte racial e, embora os programas de transferência de renda destinados a eliminar a extrema pobreza não tivessem, inicialmente, a focalização na raça, não teria como alcançar o objetivo de superação da pobreza, sem rebater sobre a população negra.

O IPEA (2015) apresenta que a taxa de analfabetismo entre as mulheres a partir de 15 anos é de $4,9 \%$ entre as brancas e 10,2\% entre negras. Dentre as adolescentes com idade entre 15 e $17 a n o s$, o atraso escolar é de $30,7 \%$ das pretas ou pardas e de $19,9 \%$ das mulheres brancas (IBGE, 2017). Se a referência for ensino superior, 23,5\% das mulheres brancas têm

\footnotetext{
1 A inclusão do "quesito cor" nos sistemas de informação e nos documentos do SUS consta no documento "Compromissos do Ministério da Saúde para com a saúde da mulher", legitimado pelo Ministro Humberto Costa, em 28 mai. 2003. BRASIL (2005, p. 16).
} 
ensino superior completo, enquanto $10,4 \%$ de mulheres pretas ou pardas concluíram o nível superior (IBGE, 2017).

Diante de tal informação, corroboramos com a ideia de que "a pobreza no Brasil tem raça/cor, sexo e etnia. Esta afirmação, interpretada por alguns como esquizofrênica, está pautada em números tão cruéis quanto seu reflexo nos corpos e nas mentes de negros e negros" (LOPES, 2005, p. 57). A situação de pobreza na qual se encontra grande parte da população brasileira é intensificada sobre a mulher negra. Tal fato, segundo Lucena (2010), caracteriza a "feminização da pobreza", tendo em vista as condições de vida, ponderando que os desfavoráveis fatores socioeconômicos e educacionais contribuem para que a mulher negra seja mais suscetível a algumas doenças, reafirmando o que revelam algumas pesquisas ${ }^{2}$ realizadas no Brasil, que explicitam a existência de uma diferença enorme nas condições de viver, adoecer e morrer, dependendo da raça/cor da população.

Inclusive torna-se necessário elucidar que, segundo Brasil (2010), há um consenso entre os estudiosos acerca das doenças que mais acometem a população negra brasileira. As categorias geralmente utilizadas são as seguintes:

a) geneticamente determinados: doença falciforme, deficiência de glicose 6-fosfato deficiência de glicose 6-fosfato desidrogenase, foliculite;

b) adquiridos em condições desfavoráveis - desnutrição, anemia ferropriva, doenças do trabalho, DST/HIV/aids, mortes violentas, mortalidade infantil elevada, abortos sépticos, sofrimento psíquico, estresse, depressão, tuberculose, transtornos mentais (derivados do uso abusivo de álcool e outras drogas);

c) de evolução agravada ou tratamento dificultado - hipertensão arterial, diabetes melito, coronariopatias, insuficiência renal crônica, câncer, miomatoses. (BRASIL, 2010, p. 15).

Levando em consideração a amplitude do quadro apresentado acima sobre as particularidades apresentadas em torno da saúde de negras e negras brasileiras (as), restringiremos a discussão às doenças mais conhecidas.

Originária entre as populações africanas, as anemias falciformes foram trazidas para o Brasil juntamente com as negras e os negros escravizados. "A doença é hereditária e muito comum entre a população negra brasileira, cerca de 1 em cada 8 afro-brasileiro tem o chamado traço falcêmico" Lucena (2010) apud Rocha (2001). Destaque para o dado que nos

\footnotetext{
${ }^{2}$ Ver Lopes (2005); (2008); Lopes e Werneck (2009); Oliveira (2003).
} 
revela que em 2013, 77\% dos nascidos vivos em Pernambuco eram de mães negras (SANTOS, 2015).

As Mulheres com anemia falciforme apresentam maior risco de abortamento e complicações durante o parto, por ser uma doença de maior prevalência entre as negras: torna-se evidente que elas necessitam de um acompanhamento pré-natal mais intensivo.

A origem racial e predomínio entre negros e mulatos é um aspecto de significativa importância quando se considera a doença do ponto de vista de saúde coletiva e se pretende estabelecer estratégias para seu controle. A reconhecida heterogeneidade dos diferentes estratos sociais e econômicos no país, com predomínio de negros nos grupos mais pobres e menos educados, em especial na periferia dos grandes centros urbanos, tornam estas doenças mais comuns nestes grupos sociais. Portanto, estratégias que visem ao controle das doenças falciformes, para serem eficientes, devem estar associadas à melhoria das condições de higiene, saúde pública e educação destes focos de miséria (OLIVEIRA, 2001, p. 127-128).

Outra informação trazida por Santos (2015) nos mostra que $82,9 \%$ dos nascidos vivos em 2013 eram de mães negras na faixa de 10 a 19 anos, informação que traz ainda o grande volume de gravidez na adolescência, característica que, em si, já configura a gravidez de risco. A pesquisadora sinaliza também que a proporção de mães adolescentes negras é mais de 5 vezes superior à de mães adolescentes brancas. E do total de mulheres negras gestantes, apenas 55\% realizam as sete consultas de pré-natal recomendadas pela Organização Mundial de Saúde/OMS.

Ao cruzar as informações, dados dos nascidos vivos e a escolaridade das mães, o estudo revelou que, dentre as mães com nenhuma escolaridade, as negras representaram $87,6 \%$ e $10,7 \%$ de mães brancas. Ao analisar o grupo com 12 anos ou mais de escolaridade, a diferença é de $51,4 \%$ nascidos de mães negras e 48,0\% de mães brancas. Esse dado nos leva a refletir que as mulheres com maior escolaridade têm mais acesso a informações e mecanismos de contracepção, tendo, portanto, uma vida sexual e reprodutiva relativamente mais saudável (SANTOS, 2015).

De acordo com a OMS, a morte materna é caracterizada por óbito no período de gestação e até 42 dias após o termino da gestação: nesse conceito também estão incluídas as mortes causadas por aborto espontâneo ou inseguro. Ao considerar o recorte cor, Brasil (2005) revela que

as causas de morte materna estão relacionadas à predisposição biológica das negras para doenças como a hipertensão arterial, fatores relacionados à 
dificuldade de acesso e à baixa qualidade do atendimento recebido e a falta de ações e capacitação de profissionais de saúde voltadas para os riscos específicos aos quais as mulheres negras estão expostas (BRASIL, 2005, p. $11)$.

Martins (2006) destaca que, embora existam pesquisas sobre a mortalidade materna, e embora até reconheça que o interesse pelo assunto parece ter aumentado entre os pesquisadores, ainda são escassas as pesquisas que apresentam o recorte étnico/racial. A autora destaca também que, muitas vezes, o "quesito cor" não é preenchido na Declaração de Óbito.

O Relatório Socioeconômico da Mulher/RASEAM (2014) da Secretaria de Políticas para Mulheres da Presidência da República (SPM-PR), informa que, em 2013, 62,8\% das mortes decorrentes de gravidez atingem mulheres negras e $35,6 \%$ mulheres brancas. $O$ relatório afirma que, no período entre 2000 e 2012, as mortes por hemorragia entre as mulheres brancas caíram de 141 casos por 100 mil partos para 93 casos; entre as mulheres negras, aumentou de 190 para 202. As mortes de mulheres brancas por aborto caíram de 39 para 15 por 100 mil partos; entre as negras, aumentou de 34 para 51.

No estado pernambucano, os índices se revelam diferentes. Santos (2015) elucida de acordo com informações da Secretaria Estadual de Saúde (com dados do Sistema de Informação de Mortalidade do Ministério da Saúde). Entre os anos de 2006 e 2013, as mortes entre mulheres negras caíram de $73,6 \%$ para $69,5 \%$ do total de mortes maternas. Já entre as brancas, houve um aumento de $21,8 \%$ para $28 \%$, no mesmo período. Observa-se que, mesmo tendo uma pequena queda, os dados ainda assim são alarmantes.

Surgida nos anos de 1980, a AIDS, uma doença atribuída a um "grupo de risco", teve naquele momento a relação de infectados de 25 homens para 1 mulher infectada; na década de 1990, com sua epidemia, a doença sofreu uma maior interiorização, assim como seu alastramento entre os mais pobres. Acrescenta-se a isso um crescimento de casos entre as mulheres - denominado de feminização da epidemia.

No ano 2000, essa proporção chegou a 1 mulher a cada 2 homens (DANTAS, 2003). Ainda segundo mesmo autor, no início dos anos 1990, os estudos revelaram que o padrão de contaminação passava por grandes mudanças: novos casos de Aids cresciam entre a população mais pobre, sendo esta, hoje, a mais afetada. Ao mencionar a população negra, temos que, até 2002, os dados do Ministério da Saúde não notificavam raça/etnia, 
dificultando políticas dirigidas para essa população, como também para a população indígena (BRASIL, 2003).

De acordo com dados do Ministério da Saúde (2012), a contaminação das mulheres ocorre, majoritariamente, através de relações heterossexuais, e a maioria dessas mulheres infectadas vive com parceiros fixos. O RASEAM 2014 informa ainda que vem aumentando o número de mulheres diagnosticadas com HIVIAIDS na faixa etária de 35 a 39 anos. As taxas de mortalidade mais altas estão nas Regiões Sul, Sudeste e Norte, com 6,1 mortes, 4,4 e 4,3, respectivamente.

O estudo realizado por Santos (2015) revela que, em Pernambuco, no período entre 2002 e 2013, o número de mulheres negras contaminadas passou de $64,3 \%$ para $82,3 \%$. Entre as brancas, esse percentual caiu de $34,8 \%$ para $16,8 \%$, no mesmo período. Entre os homens, também ocorreram diferenças entre o número de contaminados: entre os homens brancos, o percentual caiu de $27,1 \%$ para $22,2 \%$ e, entre os negros, subiu de $72,9 \%$ para $77,3 \%$ (SANTOS, 2015).

O Diabetes mellitus é outra doença que tem grande incidência sobre a população negra. Segundo Brasil (2005), as mulheres negras têm 50\% a mais de chances de desenvolver diabetes que as brancas e a situação se agrava porque na população diabética, a hipertensão arterial é duas vezes maior que na população geral. Mulheres diabéticas estão mais expostas à gravidez de alto risco. A hipertensão arterial representa $2 / 3$ das causas de morte materna entre as mulheres negras (PNUD, 2001). A OMS apud Lucena (2010, p. 108) afirma: "esta é a doença crônica que mais mata o mundo contemporâneo".

Tornou-se evidente que os fatores sociais, políticos, culturais e econômicos influenciam sob a condição de saúde da população e ao referir-se aos negros (as), "o meio ambiente que exclui e nega o direito natural de pertencimento determina condições especiais de vulnerabilidade" (LOPES, 2005, p. 55), pois,

Além da inserção social desqualificada, desvalorizada (vulnerabilidade social) e da invisibilidade de suas necessidades reais nas ações e programas de assistência, promoção de saúde e prevenção de doenças (vulnerabilidade programática), mulheres e homens negros vivem em um constante estado defensivo. Essa necessidade infindável de integrar-se e, ao mesmo tempo, proteger-se dos efeitos adversos da integração, pode provocar comportamentos inadequados, doenças psíquicas, psicossociais e físicas (vulnerabilidade individual). 
Ou seja, as vulnerabilidades as quais a população negra está sujeita ocorrem em razão das desigualdades sociais existentes na estrutura de nossa sociedade, que estão inevitavelmente relacionadas ao racismo, à classe social e, no caso das mulheres, também ao sexismo.

Contudo, é relevante destacar o processo de luta do povo negro, que, apesar de todas as dificuldades, conquistas foram alcançadas, muitos participaram ativamente de processos históricos relevantes, dentre os quais destaca-se a forte participação no Movimento de Reforma Sanitária e a 8a Conferência Nacional de Saúde onde foi garantido que o acesso à saúde seria um direito universal e dever do Estado.

No cenário internacional, destaca-se a participação na III Conferência Mundial de Combate ao Racismo, Discriminação Racial, Xenofobia e Intolerância Correlata, ocorrida no ano de 2001, em Durban, na África, onde foram reivindicadas aos governantes e organismos internacionais ações efetivas no referente à equidade étnico racial (BRASIL, 2010). A criação da Secretaria Especial de Políticas de Promoção da Igualdade Racial- SEPPIR (2003), órgão criado com a atribuição institucional de promover a igualdade e a proteção dos direitos individuais e grupos raciais.

Em 2006, foi aprovada a Política Nacional de Saúde Integral da População Negra em 10 de novembro. A política reafirma os princípios do SUS e tem como marca o "reconhecimento do racismo, das desigualdades étnicos- raciais e do racismo institucional como determinantes sociais das condições de saúde, com vistas à promoção da equidade em saúde" (BRASIL, 2010).

\section{Considerações finais}

Diante dos diversos elementos apontados no presente estudo, fica evidente que para a existência de uma análise adequada das condições sociais e da saúde da população negra, é preciso levar em consideração a grave e insistente questão do racismo no Brasil. Dessa forma, ao mencionar a particularidade de gênero, verifica-se que são imperativas reflexões, bem como intervenções que abarquem as especificidades da condição da mulher negra na sociedade brasileira, historicamente desigual.

Nesse sentido, evidenciadas as particularidades e os determinantes das doenças que acometem a população negra, faz-se necessário que o direito à saúde, preconizado 
constitucionalmente, viabilize e consolide a igualdade racial, promovendo a dignidade e a justiça social. Cabe ressaltar que negar os impactos do processo de discriminação ao qual a população negra foi historicamente exposta é reproduzir as históricas posturas racistas que inviabilizam uma atenção consistente à saúde dessa população.

Cabe destacar as reivindicações permanentes das organizações de mulheres negras pelo estabelecimento de estratégias de enfrentamento ao racismo institucional na Saúde, entendido como determinante na qualidade do atendimento que é oferecido à população negra.

\section{Referências}

BRASIL. Ministério da Saúde. Perspectiva da equidade no pacto nacional pela redução da mortalidade materna e neonatal: atenção à saúde das mulheres negras / Maria Auxiliadôra da Silva Benevides et al. 2005 .

BRASIL. Ministério da Saúde. Secretaria de Gestão Estratégica e Participativa. Departamento de Apoio à Gestão Participativa. Política Nacional de Saúde Integral da População Negra: uma política para o SUS / Ministério da Saúde, Secretaria de Gestão Estratégica e Participativa, Departamento de Apoio à Gestão Participativa. - Brasília: Editora do Ministério da Saúde, 2010.

BRASIL. Presidência da República. Secretaria de Políticas para as Mulheres. Relatório Anual Socioeconômico da Mulher. Brasília, mar. 2015.

CRENSHAW, kimberlé. Documento para o encontro de Especialistas em aspectos da Discriminação racial Relativos ao gênero. In: Estudos Feministas. 2002. Disponível em: http://www.scielo.br/pdf/ref/v1on1/11636.pdf. Acesso em: 10 abr. 18.

CUNHA, Estela Maria Garcia de Pinho da. Recorte étnico-racial: caminhos trilhados e novos desafios. In: WERNECK, Jurema; BATISTA, Luís Eduardo; LOPES, Fernanda (Org.). Saúde da população negra. Petrópolis: DP et Al; Brasília: ABPN, 2012. 328p. (Negras e negros: pesquisa em debates).

DANTAS, S. M. Rocha S. Igualdade de gênero e HIVIAIDS: uma política por construir. Rede Feminista de Saúde - Rede Nacional Feminista de Saúde Direitos Sexuais e Direitos Reprodutivos e UNIFEM - United Nations Development Fund for Women; 2003. Disponível em: http://www.redesaude.org.br/Homepage/Cartilhas/Igualdade\%2ode\%2oG\%EAnero\%2oe\%2oHIV\% 20Aids\%2ouma\%2opol\%EDtica\%2opor\%2oconstruir.pdf. Acesso em: 14mar. 2018.

INSTITUTO BRASILIEIRADE GEOGRAFIAEESTATÍSTICA(IBGE). Estatísticas de Gênero: Indicadores sociais das mulheres no Brasil. Brasília, 2018. Disponível em:

https://biblioteca.ibge.gov.br/visualizacao/livros/liv101551_informativo.pdf. Acesso em: 05 abr. 2018.

INSTITUTO BRASILIEIRADE GEOGRAFIAEESTATÍSTICA(IBGE). População chega a 205,5 milhões, com menos brancose mais pardose pretos. Brasília, 24 nov. 2017. Última Atualização: 27 nov. 2017, 11h26. Disponível em: https://agenciadenoticias.ibge.gov.br/agencia-noticias/2012-agencia-denoticias/noticias/18282-pnad-c-moradores.html. Acesso em: 07 abr. 2018. 
LOPES, F. Para além da barreira dos números: desigualdades raciais e saúde. Cadernos de Saúde Pública. Rio de Janeiro, v. 21, n. 5, 2005.

LOPES, F.; WERNECK, J. Saúde da População Negra: da conceituação às políticas públicas de direito. In: WERNECK, J. (Org.). Mulheres Negras: um olhar sobre as lutas sociais e as políticas públicas no Brasil. Rio de Janeiro: Criola, 2009. p. 5-23. Disponível em: <http://www.criola.org.br/ livro_mulheresnegras.pdf>. Acesso: 13 mai. 2018.

LOPES, Fernanda. Experiências desiguais ao nascer, viver, adoecer e morrer: tópicos em saúde da população negra no Brasil. In: BRASIL. Ministério da Saúde. Secretaria Especial de Políticas de Promoção da Igualdade Racial. Caderno de textos básicos do seminário nacional de saúde da população negra. Brasília, 2005. p. 39-100.

LUCENA, Maria de Fátima Gomes de. Saúde, Gênero e Violência: Um Estudo Comparativo Brasil/França Sobre A Saúde da mulher negra. Recife: Ed. Universitária da UFPE, 2010.

MARTINS, Alaerte Leandro. Mortalidade materna de mulheres. Cad. Saúde Pública. Rio de Janeiro, v. 22, n. 11: 2, p. 473-2479, nov. 2006. Disponivel em: http://www.scielo.br/scielo.php?pid=S0102311X2006001100022\&script=sci_arttext Acesso em: 20 ago. 2012.

MESSIAS, Ana Elisa Araújo. A saúde da mulher: a violência como problema de saúde pública e a importância do recorte étnico/racial. Revista África e Africanidades. [S. I.], ano I, n. 2, ago. 2008. Disponivel em: www.africaeafricanidades.com Acesso em: 28 jul. 2011.

MUNANGA, K. Uma abordagem conceitual das noções de raça, racismo, identidade e etnia. In: Programa de Educação sobre o Negro na Sociedade Brasileira. Niteroí: Eduff, 2000. Disponível em: http://www.uff.br/penesb/images/publicacoes/Penesb\%205\%20-

\%20Texto\%2oKabenguele\%2oMunanga.pdf. Acesso em: 08 abr. 2018.

NASCIMENTO, Silvana Silva do. RAIMUNDO, Valdenice José. A política de Assistência Social. A mulher Negra e os Programas de Transferência de Renda. In: $19^{\circ}$ Redor.

NASCIMENTO, Silvana Silva do; RODRIGUES, Michelle de Lima; SILVA, Rúbia de Kássia Alexandre e. Saúde da Mulher e a Tripla Discriminação Sofrida Pelas Negras no Brasil. [S. I., S; d.]. Disponível em: http://www.itaporanga.net/genero/3/08/22.pdf. Acesso em: 10 mar. 2018.

OLIVEIRA, Fátima. Saúde da População Negra: Brasil ano 2001. Brasília: Organização PanAmericana da Saúde, 2003. Disponível em: <http://www.opas.org.br/sistema/arquivos/o081.pdf >. Acessado em 15 mar. 2018.

Programa das Nações Unidas para o Desenvolvimento (PNUD) et al. Subsídios para o debate sobre a Política Nacional de Saúde da População Negra: Uma Questão de Equidade. Documento resultante do Workshop Interagencial de Saúde da População Negra, Brasília, 6, 7 dez. 2001. PNUD/OPAS/DFID/ UNFPA/UNICEF/UNESCO/UNDCP/UNAIDS/ UNIFEM. Disponivel em : http://bvsms.saude.gov.br/bvs/publicacoes/saudepopnegra.pdf. Acesso em: 06 abr. 2011.

SANTOS, Gevanilda. A cultura Política da Negação do Racismo Institucional. Racismo no Brasil: Percepções da discriminação e do preconceito no século XXI. São Paulo: Editora Fundação Perseu Abramo, 2005.

SANTOS, Sony. Saúde. Condições De Vida Das Mulheres Negras Em Pernambuco. Recife, Sos Corpo - Instituto Feminista para a Democracia, 2015. Disponível em: http://soscorpo.org/wp- 
content/uploads/Condicoes-de-Vida-das-Mulheres-Negras-em-Pernambuco.pdf. Acesso em: 01 fev. 2018.

WERNECK, J; MENDONÇA, M.; WHITE, E.C. O livro da Saúde das Mulheres Negras: nossos passos vêm de longe. 2. ed. Rio de Janeiro: Pallas/Criola, 2002.

Silvana Silva do Nascimento

Especialista em Urgência, Emergência e Trauma na modalidade de Residência em Saúde/UPE; Especialista em Gestão da Política de Assistência Social/UNICAP; Graduada em Serviço Social/UFPE. E-mail: silvana_nsilva@hotmail.com 